\title{
Signatures of a Quantum Griffiths Phase Close to an Electronic Nematic Quantum Phase Transition
}

\author{
Pascal Reiss $\odot,{ }^{1, *}$ David Graf, ${ }^{2}$ Amir A. Haghighirad $\odot,{ }^{1,3}$ Thomas Vojta ${ }^{4},{ }^{4}$ and Amalia I. Coldea ${ }^{1, \dagger}$ \\ ${ }^{1}$ Clarendon Laboratory, Department of Physics, University of Oxford, Oxford OX1 3PU, United Kingdom \\ ${ }^{2}$ National High Magnetic Field Laboratory, Florida State University, Tallahassee, Florida 32310, USA \\ ${ }^{3}$ Institute for Quantum Materials and Technologies, Karlsruhe Institute of Technology, 76021 Karlsruhe, Germany \\ ${ }^{4}$ Department of Physics, Missouri University of Science and Technology, Rolla, Missouri 65409, USA
}

(Received 4 December 2020; accepted 8 October 2021; published 9 December 2021)

\begin{abstract}
In the vicinity of a quantum critical point, quenched disorder can lead to a quantum Griffiths phase, accompanied by an exotic power-law scaling with a continuously varying dynamical exponent that diverges in the zero-temperature limit. Here, we investigate a nematic quantum critical point in the ironbased superconductor $\mathrm{FeSe}_{0.89} \mathrm{~S}_{0.11}$ using applied hydrostatic pressure. We report an unusual crossing of the magnetoresistivity isotherms in the nonsuperconducting normal state that features a continuously varying dynamical exponent over a large temperature range. We interpret our results in terms of a quantum Griffiths phase caused by nematic islands that result from the local distribution of Se and S atoms. At low temperatures, the Griffiths phase is masked by the emergence of a Fermi liquid phase due to a strong nematoelastic coupling and a Lifshitz transition that changes the topology of the Fermi surface.
\end{abstract}

DOI: 10.1103/PhysRevLett.127.246402

Introduction.-A central characteristic of finite- and zero-temperature phase transitions is how the spatial and temporal correlation lengths evolve as the transition is approached. For clean and continuous phase transitions, scaling theory predicts power-law divergences of both correlation lengths as a function of control parameter, with the critical exponents reflecting the universality class. Moreover, the spatial and temporal correlation lengths are closely related by the dynamics of the system [1-4]. In the presence of quenched disorder, this relation may be lost. Quenched disorder is perfectly correlated in time, but can harbor a spatially varying order parameter. In this situation, a smeared phase transition can occur, where ordered islands form within a disordered bulk $[5,6]$. Moreover, when order parameter fluctuations within the islands are non-negligible, a Griffiths phase can emerge, which leads to continuously varying critical exponents as a function of temperature and control parameter, fundamentally different to clean systems [7-13].

Experimentally, quantum Griffiths phases have been identified in ferromagnetic alloys $[14,15]$ and superconducting thin films [16-19] and they are being discussed in the context of heavy fermions $[20,21]$ and the hidden-order phase of $\mathrm{URu}_{2} \mathrm{Si}_{2}$ [22]. In the thin-film systems, sharp crossings of the magnetoresistivity isotherms have emerged as a distinctive experimental signature [23-26]. A scaling analysis revealed a temperature-dependent critical exponent $z \nu$ that diverges in the low-temperature limit. This is a hallmark of a quantum Griffiths phase ( $\nu$ is the critical correlation length exponent, and $z$ the dynamical exponent) [8-13].
In this Letter, we report the experimental realization of an unconventional quantum Griffiths phase in an electronic nematic system. Specifically, we report the magnetoresistivity of the quasi-2D bulk superconductor $\mathrm{FeSe}_{0.89} \mathrm{~S}_{0.11}$ when tuned to the vicinity of its zero-temperature nematic quantum critical point (QCP) using a hydrostatic pressure of 4.7 kbar [Fig. 1(a)] [27]. Only at the nematic QCP, the magnetoresistivity isotherms show a remarkably sharp crossing at about $30 \mathrm{~T}$ over nearly two decades in temperature up to $30 \mathrm{~K}$. Scaling of the magnetoresistivity yields a critical exponent $z \nu$ which increases by more than 2 orders of magnitude and diverges at low temperatures, in agreement with the quantum Griffiths scenario. This divergence is much stronger than previous reports of non-nematic systems where a comparatively modest enhancement of $z \nu$ was observed [16-19]. We argue that the Griffiths phase is induced by the local distribution of isoelectronic Se and $\mathrm{S}$ atoms that promote the formation of nematic islands in the vicinity of the nematic QCP, as shown in Figs. 1(b) and 1(c). Below a crossover temperature $T \approx 10 \mathrm{~K}$, the quantum Griffiths phase and the nematic QCP appear to be masked by an emergent nonzero energy scale which coincides with the reentrance of Fermi liquid behavior attributed to a strong nematoelastic coupling, as well as a topological Lifshitz transition of the Fermi surface [27-29].

Methods.-Single crystals of $\mathrm{FeSe}_{1-x} \mathrm{~S}_{x}$ with $x=0.11$ sulfur substitution were grown using the $\mathrm{KCl} / \mathrm{AlCl}_{3}$ chemical vapor transport method as described elsewhere [30]. High-pressure, high-field measurements for samples $\mathrm{B}$ and $\mathrm{C}$ were carried out in the $45 \mathrm{~T}$ hybrid DC facility in 


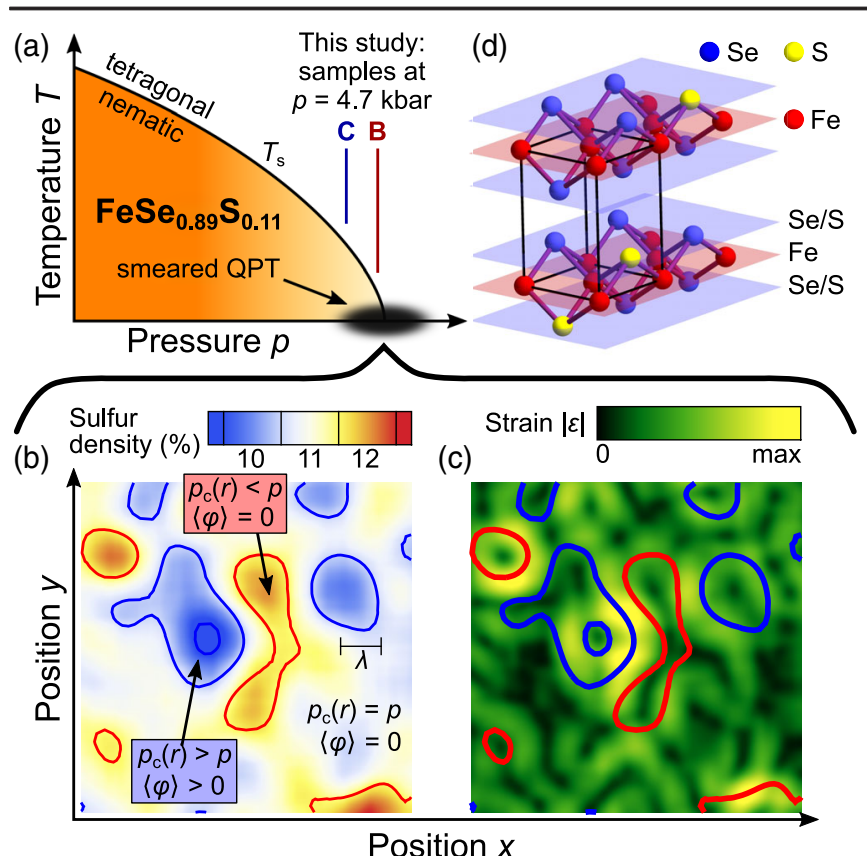

Position $x$

FIG. 1. (a) Pressure-temperature phase diagram and (d) crystal structure of $\mathrm{FeSe}_{0.89} \mathrm{~S}_{0.11}$. The relative position of samples $\mathrm{B}$ and $\mathrm{C}$ under a pressure of $p=4.7 \mathrm{kbar}$ are indicated by vertical lines. (b),(c) The spatial distribution of small $\mathrm{S}$ atoms induces locally varying critical pressures $p_{c}(r)$ and random local strains. Close to the nematic quantum phase transition (QPT), this leads to the formation of nematic islands. The scale shows the experimental mean free path length $\lambda$ [27].

Tallahassee. We used Daphne Oil 7575 as pressure medium which ensures hydrostatic conditions for much higher pressures than reported here, and we used the ruby fluorescence shifts below $4 \mathrm{~K}$ to determine the pressure. Low-field measurements up to $13.5 \mathrm{~T}$ were carried out on sample A in a QuantumDesign PPMS in Oxford. Here, Daphne Oil 7373 was used, and the pressure was determined by the superconducting transition of tin. Samples were aligned with the magnetic field parallel to the crystallographic $c$ axis to avoid breaking an in-plane symmetry. Transport measurements were performed using a standard 4 or 5 contact setup, using the AC LockIn technique with a low frequency $f \approx 20 \mathrm{~Hz}$, and a low excitation current $I_{p}=1 \mathrm{~mA}$ within the $(a b)$ plane.

Results.-Figures 2(a) and 2(b) show the temperature dependence of the magnetoresistivity of two different single crystals $\mathrm{B}$ and $\mathrm{C}$ of $\mathrm{FeSe}_{0.89} \mathrm{~S}_{0.11}$ under a hydrostatic pressure of $p=4.7 \mathrm{kbar}$, which are in the immediate vicinity of their nematic QCPs $\left[p_{c}=4.8(3) \mathrm{kbar}\right.$ for sample B and 5.2(3) kbar for sample C, respectively as shown in Fig. 1(a) and in the Supplemental Material [27,31,39] ]. All magnetoresistivity isotherms cross around a similar magnetic field, $\mu_{0} H^{\star} \approx 28.6 \mathrm{~T}$ for sample $\mathrm{B}$ and $28.0 \mathrm{~T}$ for sample $\mathrm{C}$, with similar resistivities $\rho^{\star} \approx 32$ and $34 \mu \Omega \mathrm{cm}$, respectively. This crossing occurs over nearly two decades in temperature $0.3 \mathrm{~K} \lesssim T \lesssim 30 \mathrm{~K}$ and its
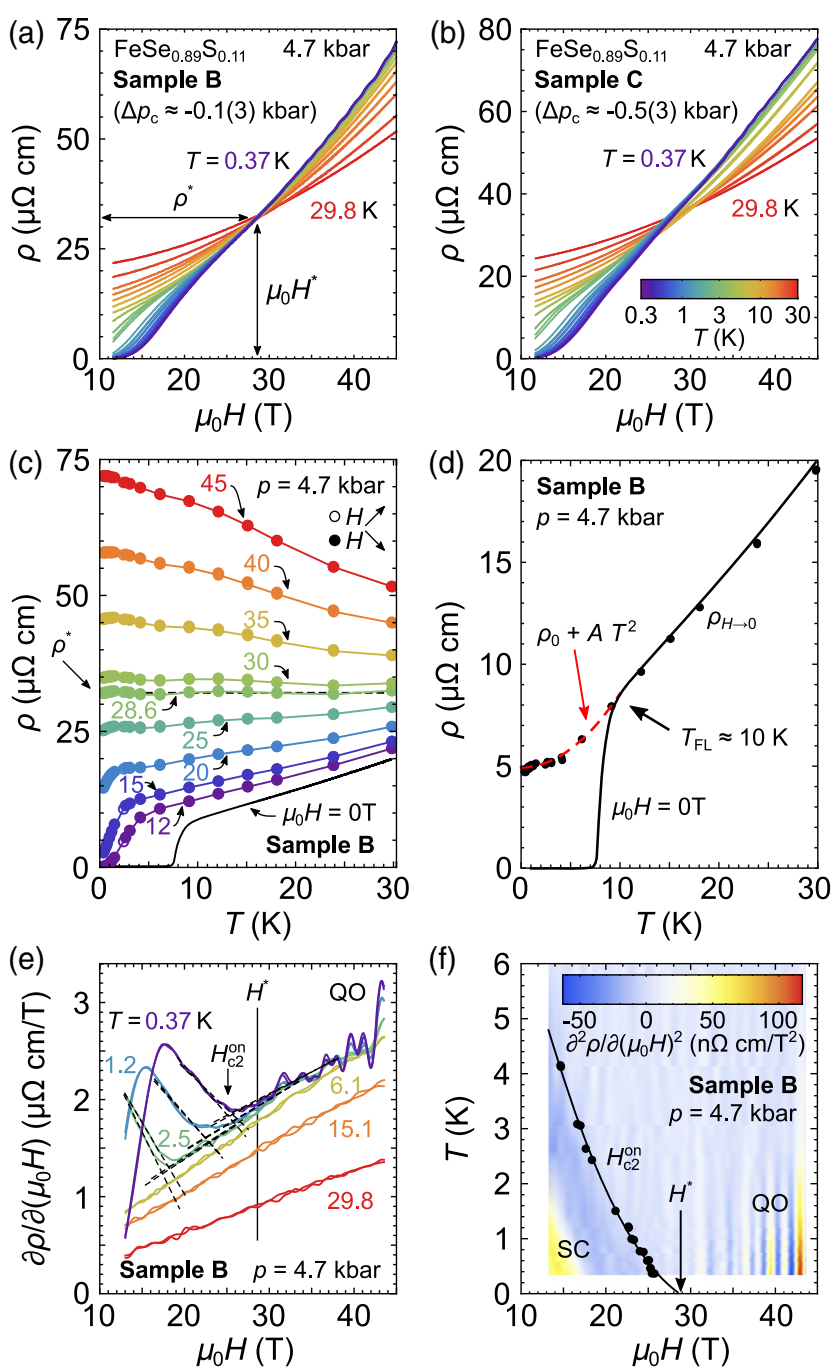

FIG. 2. (a),(b) The isothermal magnetoresistivity of samples B and $\mathrm{C}$ at a pressure of $p=4.7 \mathrm{kbar}$ cross at $\mu_{0} H^{\star} \approx 28 \mathrm{~T}$, $\rho^{\star} \approx 33 \mu \Omega \mathrm{cm}$. Up and down sweeps show no hysteresis. The pressure difference in brackets represents the distance to the critical pressure [see Fig. 1(a) and in the Supplemental Material [31]]. (c) The same data as in panel (a), but as a function of temperature in fixed field. (d) The actual and extrapolated zerofield resistivities, $\rho(T)$ (solid line) and $\rho_{H \rightarrow 0}$ (points, see the Supplemental Material [31]). Error bars are smaller than the symbol size. The red dashed line is a fit to Fermi liquid behavior ( $\rho_{0} \approx 4.8 \mu \Omega \mathrm{cm}, A \approx 0.038 \mu \Omega \mathrm{cm} / \mathrm{K}^{2}$ ). (e) The first derivative reveals the onset of superconductivity, indicated by arrows. Large quantum oscillations (QO) can be seen for $H>H^{\star}$. (f) The extrapolation of the superconducting (SC) onset coincides with $H^{\star}$ only at $T=0$. All reported data are measured at $p=4.7$ kbar.

significance becomes evident in the resistivity plots as a function of temperature in constant field, shown in Fig. 2(c). For $H<H^{\star}$, the resistivity follows a metallic-like behavior with $\partial \rho / \partial T>0$ before the sample becomes superconducting below $T_{c}^{\mathrm{on}} \approx 10 \mathrm{~K}$ [Fig. 2(d)]. Equivalently, the onset magnetic field, $H_{c 2}^{\text {on }}$, between the superconducting and 
normal phases can be identified in magnetic fields smaller than $H^{\star}$ in Fig. 2(e) whose zero-temperature extrapolation coincides with $H^{\star}$ [Fig. 2(f)]. Thus, the magnetoresistivity crossing occurs strictly within the non-superconducting normal phase for all finite temperatures $\left[H^{\star}>H_{c 2}^{\text {on }}(T)\right]$, implying that this behavior describes the normal phase in the vicinity of the nematic QCP.

In high magnetic fields above $H^{\star}$, the resistivity shows insulatinglike behavior $(\partial \rho / \partial T<0)$, before it saturates below $T \approx 2 \mathrm{~K}$ [Fig. 2(c)], similar to previous reports [40]. Despite this insulatinglike behavior, the large magnetoresistivity is a feature of the metallic, compensated multiband system $\mathrm{FeSe}_{1-x} \mathrm{~S}_{x}[29,40,41]$. Quantum oscillations are visible for temperatures below $\approx 5 \mathrm{~K}$ [Fig. 2(e)], demonstrating the existence of a Fermi surface and highlighting the high quality of the samples [27,42]. A twoband analysis of the magnetoresistivity allows us to extrapolate the zero-field resistivity from high magnetic fields [31], which indicate Fermi liquid behavior below a crossover temperature $T_{\mathrm{FL}} \approx 10 \mathrm{~K}$, shown in Fig. 2(d) $[27,40]$. The orbitally averaged effective masses from quantum oscillations show nondivergent electronic correlations in the vicinity of the nematic QCP, as discussed in detail in Ref. [27], likely due to a coupling between the nematic order parameter and the lattice [28,43-45].

Next, we use a prototypical power-law scaling ansatz to describe the magnetoresistivity of $\mathrm{FeSe}_{0.89} \mathrm{~S}_{0.11}$, previously applied in thin-film materials, including dirty films of FeSe [16-19,23-26]. In $d$ dimensions, the scaling is given by

$$
\rho(H, T) / \rho^{\star}=T^{(2-d) / z} f\left(\mu_{0}\left|H-H^{\star}\right| / T^{1 / z \nu}\right),
$$

with $f(0)=1$ and the critical exponent $z \nu$ [46]. Clearly, a crossing of the magnetoresistivity isotherms at a finite $\rho^{\star}$ is only possible for a two-dimensional system. Indeed, $\mathrm{FeSe}_{1-x} \mathrm{~S}_{x}$ have strongly two-dimensional electronic and superconducting properties [27,29,47-50].

In the case of a typical QCP, $z \nu$ is a constant given by the appropriate universality class, which would lead to a constant slope in Fig. 3(a) (see also the Supplemental Material [31]). This is evidently not the case here where we identify a power-law dependence of $z \nu(T) \sim T^{\alpha}$, with nonuniversal exponents $\alpha \approx-1.5$ for sample $\mathrm{B}$ and $\approx-1.0$ for sample $\mathrm{C}$, as shown in Fig. 3(b). Using this extracted $z \nu(T)$, all magnetoresistivity data collapse onto a single curve for both samples, reflecting the form of the scaling function $f$, shown in Fig. 3(c). Deviations for this scaling only occur for the superconducting transition at lowest fields and temperatures, and at the highest temperatures and fields. These deviations indicate the limits of the scaling relation, as shown in the Supplemental Material [31].

This scaling analysis reveals an interesting and unexpected feature. While a zero-temperature divergence of the effective critical exponent $z \nu(T)$ is a key signature of quantum Griffiths phases, the power-law divergence
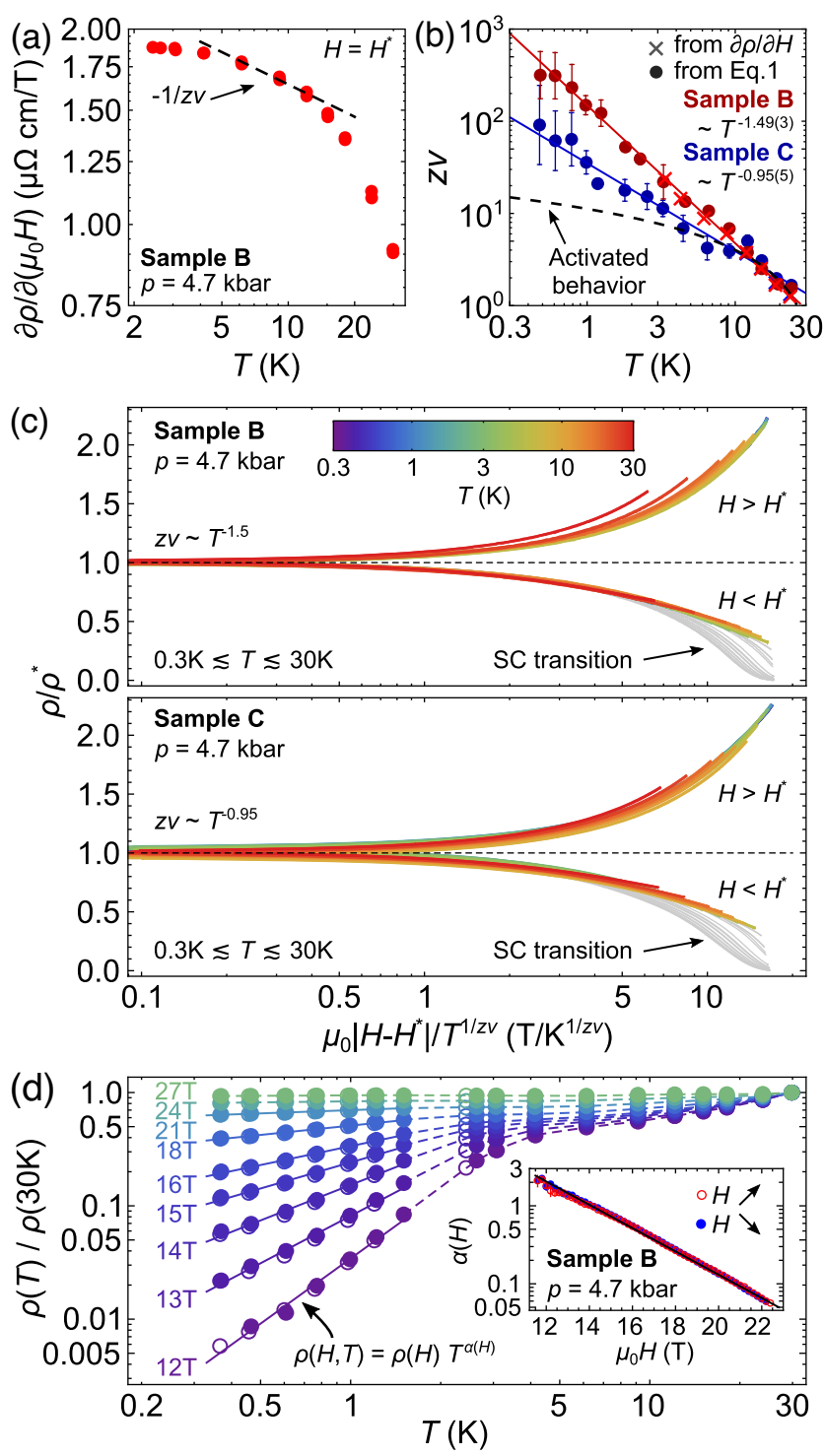

FIG. 3. (a) Log-log plot of the first derivative at the crossing field $H^{\star}$ [refer to Fig. 2(e)]. Error bars are smaller than the symbol size. The slope of the dashed line corresponds to $1 / z \nu(T)$. (b) Temperature dependence of $z \nu$ extracted from panel A (crosses) and from the piecewise extraction shown in the Supplemental Material (dots) [31]. Error bars indicate a $1 \sigma$ confidence interval. (c) Scaled magnetotransport data using $z \nu \sim$ $T^{-1.5}$ (sample B) and $z \nu \sim T^{-0.95}$ (sample C). The superconducting transitions (SC) deviate from this scaling form. (d) The lowfield, low-temperature resistivity in the mixed state follows a power-law form $\rho(H, T)=\rho(H) T^{\alpha}$. The inset shows a nearly exponential decay of $\alpha(H)$. For $\mu_{0} H>22 \mathrm{~T}$ the analysis becomes unreliable, and for $\mu_{0} H>28 \mathrm{~T}$, the exponent turns negative. All reported data are measured at $p=4.7$ kbar.

observed here is much stronger than the logarithmic divergence (activated behavior) predicted within the infiniterandomness criticality scenario [13,18,31], as shown in Fig. 3(b). In fact, a power-law divergence of $z \nu(T)$ is incompatible with the presence of a typical QCP because 

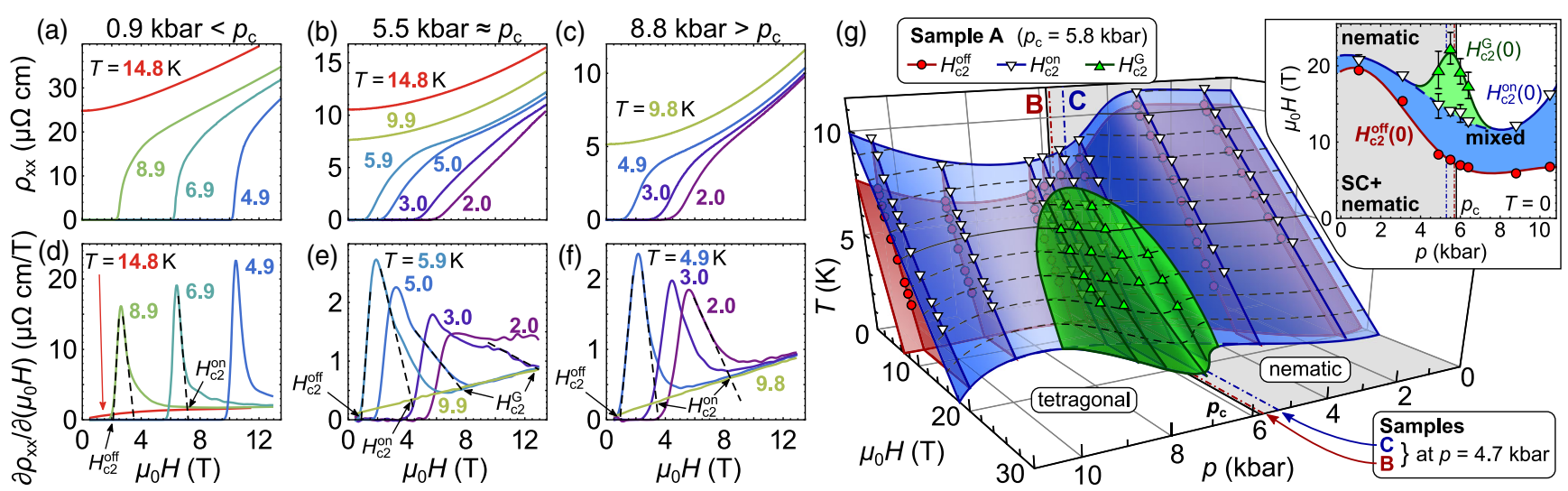

FIG. 4. Evolution of superconductivity for sample A. (a)-(c) Magnetoresistivity and (d)-(f) the corresponding first derivatives showing the development of the superconducting transition in magnetic fields for pressures across the nematic quantum phase transition. (g) Three-dimensional $H-p-T$ superconducting phase diagram. The inset shows the extrapolated critical fields, $H_{c 2}^{\text {off }}, H_{c 2}^{\text {on }}$, and $H_{c 2}^{\mathrm{G}}$, defined in panels (d)-(f), in the zero-temperature limit. The relative positions of samples B and $\mathrm{C}$ under a pressure of $4.7 \mathrm{kbar}$ in the phase diagram are indicated by dashed and dotted lines, respectively (see also Figs. 2 and 3). Error bars indicate a $1 \sigma$ confidence interval.

the temperature term $T^{1 / z \nu(T)}$ in Eq. (1) remains finite for $T \rightarrow 0$, which implies the persistence of a nonzero energy scale at lowest temperatures. Interestingly, we find that $z \nu$ deviates from the activated behavior dependence below $T \approx$ $5-10 \mathrm{~K}$ which coincides with a reentrance of Fermi liquid behavior, Fig. 2(b). This suggests a suppression of order parameter fluctuations due to a finite coupling with the lattice, or a dimensional crossover induced by a changing Fermi surface topology $[27,29,40,50]$.

We now focus on the nature of the underlying phases separated by $H^{\star}$. Figure 3(d) shows that for fields smaller than $H^{\star}$, the resistivity within the superconducting mixed state follows a power-law form $\rho \propto T^{\alpha(H)}$ over almost one decade in temperature. We attribute this power-law form to a disordered vortex-liquid phase that freezes into a vortex glass in the zero-temperature limit, as found in underdoped cuprates [25]. Crossing over into the high-field regime above $H^{\star}$ where quantum oscillations are present, the temperature-dependence of the resistivity suggests coexisting metallic and finite-range hopping channels, as discussed in the Supplemental Material [31].

To elucidate the origin and extent of the low-field disordered vortex phase, we investigate the pressure dependence of the superconducting to normal transitions in magnetic fields on sample A ( $p_{c} \approx 5.8$ kbar [27]). Figures 4(a)-4(f) show the magnetoresistivity and its derivative up to $13.5 \mathrm{~T}$ inside the nematic phase ( $0.9 \mathrm{kbar})$, close to the nematic quantum phase transition $(5.5 \mathrm{kbar})$ and within the tetragonal phase ( $8.8 \mathrm{kbar}$ ). In the nematic and tetragonal phases, the normal-to-superconducting transition widths are nearly temperature and field independent. In contrast, a visible broadening of the transition is found close to $p_{c}$, but only for high fields and at low temperatures, thus coinciding with the vortex-liquid phase in sample B. To quantify this additional broadening, we extract the superconducting offset and onset critical fields, $H_{c 2}^{\text {off }}$ and $H_{c 2}^{\text {on }}$, as shown in Figs. 4(d)-4(f).
Furthermore, we define a critical magnetic field $H_{c 2}^{\mathrm{G}}$, where the magnetoresistivity derivative has an additional shoulder before it returns to its high-temperature normal state background, which is observable only in the vicinity of $p_{c}$ [Fig. 4(e)]. Figure 4(g) summarizes all extracted critical fields and their zero-temperature extrapolations, see also the Supplemental Material [31]. Interestingly, the zero-temperature superconducting transition width peaks at the nematic quantum phase transition, doubling the extent of the superconducting mixed state. The width of the $H_{c 2}^{\mathrm{G}}(0)$ peak in pressure is estimated to be $\sigma_{p} \approx 0.7(2) \mathrm{kbar}$, which agrees well with an estimate for the pressure range of the quantum Griffiths phase, as discussed below. Figure 4(g) also shows that the zero-field superconducting transition does not display any similar broadening. This demonstrates that the peak in $H_{c 2}^{\mathrm{G}}$ is a low-temperature and high-field effect, ruling out effects of possible pressure inhomogeneities [31].

Discussion.-Quantum Griffiths phases were previously detected in inhomogeneous superconductor-to-insulator transitions in thin films, including FeSe [16-19,23-26]. Here, in bulk $\mathrm{FeSe}_{0.89} \mathrm{~S}_{0.11}$, the situation is very different. The scaling relation only describes the normal state resistivity and holds for magnetic fields up to $45 \mathrm{~T}$ and temperatures up to $30 \mathrm{~K}$, vastly exceeding the bulk superconducting phase. We therefore propose that the quantum Griffiths phase in $\mathrm{FeSe}_{0.89} \mathrm{~S}_{0.11}$ emerges from the suppression of the nematic phase with pressure $[27,39,51]$ and the formation of rare nematic islands in a tetragonal matrix due to the random distribution of sulfur atoms (Fig. 1), as suggested before (see the Supplemental Material to Ref. [5]). To demonstrate how this can lead to a quantum Griffiths phase, we sample a random distribution of $11 \% \mathrm{~S}$ atoms over a square lattice, and average the effective sulfur density $x(r)$ over the experimental quasiparticle mean-free path length $\lambda \approx 350 \AA$ [27], as shown in Figs. 1(b) and 1(c). 
The intrinsic local variation $\Delta x(r) \approx 0.4 \%$ (std. dev.) gives rise to regions with higher (lower) $\mathrm{S}$ content which have a locally lower (higher) critical pressure $p_{c}(r)$. This is the prototypical case of random-mass disorder that smears the quantum phase transition over a region $\Delta p_{c}(r)$. By comparing the reported nematic transition temperatures from pressure and isoelectronic substitution studies, we estimate $\Delta x(r) \propto \Delta p_{c}(r) \approx 0.4 \mathrm{kbar}[29,39,40,52]$. This estimate is similar to the observed pressure range of a broadened superconducting transition, $\sigma_{p}=0.7(2)$ kbar. This suggests that the peak in $H_{c 2}^{\mathrm{G}}(0)$ occurs either due to enhanced superconducting fluctuations within the nematic islands, and/or superconducting nematic islands below the percolation threshold, which get suppressed at $H^{\star}$. These effects could also provide a favorable environment for the observed inhomogeneous superconducting vortex phase in the vicinity of the nematic QCP. Finally, we note that the spatial arrangement of the $\mathrm{S}$ atoms locally breaks the $C_{4}$ symmetry of the lattice and thus introduces random-field effects. In the two-dimensional regime, they may limit the size of the nematic domains, but for weak disorder, the corresponding breakup length is exponentially large [53].

It is rather surprising that $\mathrm{FeSe}_{0.89} \mathrm{~S}_{0.11}$ appears as a clean system where quantum oscillations can be observed at lowest temperatures, and yet signatures of a quantum Griffiths phase are detected as well. Thus, in the vicinity of the electronic nematic quantum phase transition, additional effects must be considered, such as the nematoelastic coupling that quenches the two-dimensional quantum critical nematic fluctuations below a cross-over temperature $T_{\mathrm{FL}} \approx 10 \mathrm{~K}$. As a result, Fermi liquid behavior with finite electronic correlations is restored [27,29,40,50], and the quantum Griffiths phase is cut off, leading to the overly strong divergence of $z \nu$. Additionally, a band with likely 3D character is formed due to a Lifshitz transition of the Fermi surface in the proximity of the nematic QCP $[27,29]$ which may change the effective dimensionality of the system at low temperatures.

The observation of a quantum Griffiths phase in an ironbased superconductor has a number of important implications and provides new insights into the nature of nematic quantum phase transitions. Most notably, the power-law behavior of $z \nu(T)$ could provide new insights into the dynamics of (quenched) nematic quantum fluctuations. Moreover, our study provides evidence that the quantum Griffiths phase affects the mixed state of the superconducting phase. Alternative systems to search for nematic quantum Griffiths phases are those iron-based superconductors in which nematic and tetragonal phases form over limited compositional ranges around QCPs [29,40,54,55]. Experimental probes include uniaxial strain to suppress nematic fluctuations and hence to tune Griffiths phases [56]; NMR and Raman studies to probe the essential role of lattice disorder [57]; STM studies to follow the formation of nematic islands [58]; specific heat measurements to search for predicted power-law behavior at low temperatures $[14,17,20,59]$. Thus, we hope that our results will guide further theoretical and experimental research in understanding nematic quantum Griffiths phases.

In accordance with the EPSRC policy framework on research data, access to the data will be made available from Ref. [60].

We thank R. Fernandes for insightful discussions. This work was mainly supported by the EPSRC (EP/I004475/1, EP/I017836/1) and the Oxford Centre for Applied Superconductivity. P. R. and A. A. H. acknowledge financial support of the Oxford Quantum Materials Platform Grant (EP/M020517/1). A portion of this work was performed at the National High Magnetic Field Laboratory, supported by NSF Cooperative Agreements DMR-1157490, DMR-1644779 and the State of Florida. Work in Missouri was supported by the NSF (DMR1828489). We acknowledge the financial support of the Oxford University John Fell Fund. A. I. C. acknowledges an EPSRC Career Acceleration Fellowship (EP/I004475/1) and Oxford Centre for Applied Superconductivity for financial support.

\author{
*Present address: Max-Planck Institute for Solid State \\ Research, Stuttgart, Germany. \\ p.reiss@fkf.mpg.de \\ amalia.coldea@physics.ox.ac.uk
}

[1] J. A. Hertz, Zero- and low-temperature phase transitions and the renormalization group, AIP Conf. Proc. 24, 298 (1975).

[2] J. A. Hertz, Quantum critical phenomena, Phys. Rev. B 14, 1165 (1976).

[3] A. J. Millis, Effect of a nonzero temperature on quantum critical points in itinerant fermion systems, Phys. Rev. B 48, 7183 (1993).

[4] T. Moriya, Spin Fluctuations in Itinerant Electron Magnetism, Springer Series in Solid-State Sciences Vol. 56 (Springer-Verlag, Berlin, Heidelberg, New York, Tokyo, 1985).

[5] H.-H. Kuo, J.-H. Chu, J. C. Palmstrom, S. A. Kivelson, and I. R. Fisher, Ubiquitous signatures of nematic quantum criticality in optimally doped Fe-based superconductors, Science 352, 958 (2016).

[6] T. Cui and R. M. Fernandes, Smeared nematic quantum phase transitions due to rare-region effects in inhomogeneous systems, Phys. Rev. B 98, 085117 (2018).

[7] R. B. Griffiths, Nonanalytic Behavior Above the Critical Point in a Random Ising Ferromagnet, Phys. Rev. Lett. 23, 17 (1969).

[8] D. S. Fisher, Random Transverse Field Ising Spin Chains, Phys. Rev. Lett. 69, 534 (1992).

[9] D.S. Fisher, Critical behavior of random transverse-field Ising spin chains, Phys. Rev. B 51, 6411 (1995).

[10] A. Del Maestro, B. Rosenow, M. Müller, and S. Sachdev, Infinite Randomness Fixed Point of the SuperconductorMetal Quantum Phase Transition, Phys. Rev. Lett. 101, 035701 (2008). 
[11] T. Vojta, Disorder-Induced Rounding of Certain Quantum Phase Transitions, Phys. Rev. Lett. 90, 107202 (2003).

[12] T. Vojta, Rare region effects at classical, quantum, and nonequilibrium phase transitions, J. Phys. A 39, R143 (2006).

[13] T. Vojta, Quantum Griffiths effects and smeared phase transitions in metals: Theory and experiment, J. Low Temp. Phys. 161, 299 (2010).

[14] S. Ubaid-Kassis, T. Vojta, and A. Schroeder, Quantum Griffiths Phase in the Weak Itinerant Ferromagnetic Alloy $\mathrm{Ni}_{1-x} \mathrm{~V}_{x}$, Phys. Rev. Lett. 104, 066402 (2010).

[15] R. Wang, A. Gebretsadik, S. Ubaid-Kassis, A. Schroeder, T. Vojta, P. J. Baker, F. L. Pratt, S. J. Blundell, T. Lancaster, I. Franke, J. S. Möller, and K. Page, Quantum Griffiths Phase Inside the Ferromagnetic Phase of $\mathrm{Ni}_{1-x} \mathrm{~V}_{x}$, Phys. Rev. Lett. 118, 267202 (2017).

[16] Y. Xing, H.-M. Zhang, H.-L. Fu, H. Liu, Y. Sun, J.-P. Peng, F. Wang, X. Lin, X.-C. Ma, Q.-K. Xue, J. Wang, and X. C. Xie, Quantum Griffiths singularity of superconductormetal transition in $\mathrm{Ga}$ thin films, Science 350, 542 (2015).

[17] Y. Saito, T. Nojima, and Y. Iwasa, Quantum phase transitions in highly crystalline two-dimensional superconductors, Nat. Commun. 9, 778 (2018).

[18] N. A. Lewellyn, I. M. Percher, J. Nelson, J. Garcia-Barriocanal, I. Volotsenko, A. Frydman, T. Vojta, and A. M. Goldman, Infinite-randomness fixed point of the quantum superconductor-metal transitions in amorphous thin films, Phys. Rev. B 99, 054515 (2019).

[19] Y. Liu, Z. Wang, P. Shan, Y. Tang, C. Liu, C. Chen, Y. Xing, Q. Wang, H. Liu, X. Lin, X. C. Xie, and J. Wang, Anomalous quantum Griffiths singularity in ultrathin crystalline lead films, Nat. Commun. 10, 3633 (2019).

[20] M. C. de Andrade, R. Chau, R. P. Dickey, N. R. Dilley, E. J. Freeman, D. A. Gajewski, M. B. Maple, R. Movshovich, A. H. Castro Neto, G. Castilla, and B. A. Jones, Evidence for a Common Physical Description of Non-Fermi-Liquid Behavior in Chemically Substituted $f$-Electron Systems, Phys. Rev. Lett. 81, 5620 (1998).

[21] D. Gnida, Electronic Griffiths phase and quantum interference in disordered heavy-fermion systems, Phys. Rev. B 97, 081112(R) (2018).

[22] X. Ji, Y. Zhang, X. Wang, and Y. Liu, Griffiths phase-like exponents in the hidden-order state of $\mathrm{URu}_{2} \mathrm{Si}_{2}$, J. Magn. Magn. Mater. 519, 167455 (2021).

[23] G. T. Seidler, T. F. Rosenbaum, and B. W. Veal, Twodimensional superconductor-insulator transition in bulk single-crystal $\mathrm{YBa}_{2} \mathrm{Cu}_{3} \mathrm{O}_{6.38}$, Phys. Rev. B 45, 10162 (1992).

[24] R. Schneider, A. G. Zaitsev, D. Fuchs, and H. v. Löhneysen, Superconductor-Insulator Quantum Phase Transition in Disordered FeSe Thin Films, Phys. Rev. Lett. 108, 257003 (2012).

[25] X. Shi, P. V. Lin, T. Sasagawa, V. Dobrosavljević, and D. Popović, Two-stage magnetic-field-tuned superconductorinsulator transition in underdoped $\mathrm{La}_{2-x} \mathrm{Sr}_{x} \mathrm{CuO}_{4}$, Nat. Phys. 10, 437 (2014).

[26] Y. Hsiang Lin, J. Nelson, and A. M. Goldman, Superconductivity of very thin films: The superconductor-insulator transition, Physica (Amsterdam) 514C, 130 (2015).
[27] P. Reiss, D. E. Graf, A. A. Haghighirad, W. Knafo, L. Drigo, M. Bristow, A. J. Schofield, and A. I. Coldea, Quenched nematic criticality and two superconducting domes in an iron-based superconductor, Nat. Phys. 16, 89 (2020).

[28] I. Paul and M. Garst, Lattice Effects on Nematic Quantum Criticality in Metals, Phys. Rev. Lett. 118, 227601 (2017).

[29] A. I. Coldea, S. F. Blake, S. Kasahara, A. A. Haghighirad, M. D. Watson, W. Knafo, E. Sang Choi, A. McCollam, P. Reiss, T. Yamashita, M. Bruma, S. C. Speller, Y. Matsuda, T. Wolf, T. Shibauchi, and A. John Schofield, Evolution of the low-temperature Fermi surface of superconducting $\mathrm{FeSe}_{1-x} \mathrm{~S}_{x}$ across a nematic phase transition, npj Quantum Mater. 4, 2 (2019).

[30] A. E. Böhmer, V. Taufour, W. E. Straszheim, T. Wolf, and P.C. Canfield, Variation of transition temperatures and residual resistivity ratio in vapor-grown FeSe, Phys. Rev. B 94, 024526 (2016).

[31] See Supplemental Material at http://link.aps.org/supplemental/ 10.1103/PhysRevLett.127.246402 for further experimental data not shown in the main manuscript, including further pressure points on samples B and C, and further temperatures for sample A. Additional analyses are presented which support the conclusion presented in the main manuscript, which includes Refs. [32-38].

[32] M. D. Watson, T. K. Kim, A. A. Haghighirad, S. F. Blake, N. R. Davies, M. Hoesch, T. Wolf, and A. I. Coldea, Suppression of orbital ordering by chemical pressure in FeSe $_{1-x} \mathrm{~S}_{x}$, Phys. Rev. B 92, 121108(R) (2015).

[33] S. Fey, S. C. Kapfer, and K. Phillip Schmidt, Quantum Criticality of Two-Dimensional Quantum Magnets with Long-Range Interactions, Phys. Rev. Lett. 122, 017203 (2019).

[34] N. F. Mott, Conduction in non-crystalline systems, Philos. Mag. 17, 1259 (1968).

[35] N. F. Mott, Conduction in non-crystalline materials, Philos. Mag. 19, 835 (1969).

[36] N. F. Mott, Metal-insulator transitions, Pure Appl. Chem. 52, 65 (1980).

[37] A. L. Efros and B. I. Shklovskii, Coulomb gap and low temperature conductivity of disordered systems, J. Phys. C 8, L49 (1975).

[38] A. L. Efros, Coulomb gap in disordered systems, J. Phys. C 9, 2021 (1976).

[39] L. Xiang, U. S. Kaluarachchi, A. E. Böhmer, V. Taufour, M. A. Tanatar, R. Prozorov, S. L. Bud'ko, and P.C. Canfield, Dome of magnetic order inside the nematic phase of sulfur-substituted FeSe under pressure, Phys. Rev. B 96, 024511 (2017).

[40] M. Bristow, P. Reiss, A. A. Haghighirad, Z. Zajicek, S. J. Singh, T. Wolf, D. Graf, W. Knafo, A. McCollam, and A. I. Coldea, Anomalous high-magnetic field electronic state of the nematic superconductors $\mathrm{FeSe}_{1-x} \mathrm{~S}_{x}$, Phys. Rev. Research 2, 013309 (2020).

[41] M. D. Watson, T. Yamashita, S. Kasahara, W. Knafo, M. Nardone, J. Béard, F. Hardy, A. McCollam, A. Narayanan, S. F. Blake, T. Wolf, A. A. Haghighirad, C. Meingast, A. J. Schofield, H. v. Löhneysen, Y. Matsuda, A. I. Coldea, and T. Shibauchi, Dichotomy between the Hole and Electron Behavior in Multiband Superconductor FeSe Probed by Ultrahigh Magnetic Fields, Phys. Rev. Lett. 115, 027006 (2015). 
[42] D. Shoenberg, Magnetic Oscillations in Metals (Cambridge University Press, Cambridge, 1984).

[43] X. Wang and E. Berg, Scattering mechanisms and electrical transport near an Ising nematic quantum critical point, Phys. Rev. B 99, 235136 (2019).

[44] V.S. de Carvalho and R. M. Fernandes, Resistivity near a nematic quantum critical point: Impact of acoustic phonons, Phys. Rev. B 100, 115103 (2019).

[45] L. E. Vieira, V. S. de Carvalho, and H. Freire, DC resistivity near a nematic quantum critical point: Effects of weak disorder and acoustic phonons, Ann. Phys. (Amsterdam) 419, 168230 (2020).

[46] M. P. A. Fisher, Quantum Phase Transitions in Disordered Two-Dimensional Superconductors, Phys. Rev. Lett. 65, 923 (1990).

[47] T. Terashima, N. Kikugawa, A. Kiswandhi, E.-S. Choi, J. S. Brooks, S. Kasahara, T. Watashige, H. Ikeda, T. Shibauchi, Y. Matsuda, T. Wolf, A. E. Böhmer, F. Hardy, C. Meingast, H. v. Löhneysen, M.-T. Suzuki, R. Arita, and S. Uji, Anomalous Fermi surface in FeSe seen by Shubnikovde Haas oscillation measurements, Phys. Rev. B 90, 144517 (2014).

[48] M. Bristow, A. A. Haghighirad, M. D. Watson, P. Reiss, Z. Zajicek, J. Prentice, S. J. Blundell, A. McCollam, and A. I. Coldea, Multi-band effects responsible for the upper critical fields of FeSe (to be published).

[49] L. S. Farrar, M. Bristow, A. A. Haghighirad, A. McCollam, S. J. Bending, and A. I. Coldea, Suppression of superconductivity and enhanced critical field anisotropy in thin flakes of FeSe, npj Quantum Mater. 5, 29 (2020).

[50] A. I. Coldea, Electronic nematic states tuned by isoelectronic substitution in bulk $\mathrm{FeSe}_{1-x} \mathrm{~S}_{x}$, arXiv:2009.05523.

[51] K. Matsuura, Y. Mizukami, Y. Arai, Y. Sugimura, N. Maejima, A. Machida, T. Watanuki, T. Fukuda, T. Yajima, Z. Hiroi, K. Y. Yip, Y. C. Chan, Q. Niu, S. Hosoi, K. Ishida, K. Mukasa, S. Kasahara, J.-G. Cheng, S. K. Goh, Y. Matsuda, Y. Uwatoko, and T. Shibauchi, Maximizing $T_{c}$ by tuning nematicity and magnetism in $\mathrm{FeSe}_{1-x} \mathrm{~S}_{x}$ superconductors, Nat. Commun. 8, 1143 (2017).

[52] P. Reiss, M. D. Watson, T. K. Kim, A. A Haghighirad, D. N. Woodruff, M. Bruma, S. J. Clarke, and A. I. Coldea, Suppression of electronic correlations by chemical pressure from FeSe to FeS, Phys. Rev. B 96, 121103(R) (2017).

[53] E. T. Seppälä and M. J. Alava, Susceptibility and percolation in two-dimensional random field Ising magnets, Phys. Rev. E 63, 066109 (2001).

[54] A. E. Böhmer, F. Hardy, L. Wang, T. Wolf, P. Schweiss, and C. Meingast, Superconductivity-induced re-entrance of the orthorhombic distortion in $\mathrm{Ba}_{1-x} \mathrm{~K}_{x} \mathrm{Fe}_{2} \mathrm{As}_{2}$, Nat. Commun. 6, 7911 (2015).

[55] S. Hosoi, K. Matsuura, K. Ishida, H. Wang, Y. Mizukami, T. Watashige, S. Kasahara, Y. Matsuda, and T. Shibauchi, Nematic quantum critical point without magnetism in $\mathrm{FeSe}_{1-x} \mathrm{~S}_{x}$ superconductors, Proc. Natl. Acad. Sci. U.S.A. 113, 8139 (2016).

[56] M. Ghini, M. Bristow, J. C. A. Prentice, S. Sutherland, S. Sanna, A. A. Haghighirad, and A. I. Coldea, Strain tuning of nematicity and superconductivity in single crystals of FeSe, Phys. Rev. B 103, 205139 (2021).

[57] P. Wiecki, R. Zhou, M.-H. Julien, A. E. Böhmer, and J. Schmalian, Edwards-Anderson parameter and local Ising nematicity in FeSe revealed via NMR spectral broadening, Phys. Rev. B 104, 125134 (2021).

[58] H. Zhao, H. Li, L. Dong, B. Xu, J. Schneeloch, R. Zhong, M. Fang, G. Gu, J. Harter, S. D. Wilson, Z. Wang, and I. Zeljkovic, Nematic transition and nanoscale suppression of superconductivity in $\mathrm{Fe}(\mathrm{Te}, \mathrm{Se})$, Nat. Phys. 17, 903 (2021).

[59] A. H. Castro Neto, G. Castilla, and B. A. Jones, NonFermi Liquid Behavior and Griffiths Phase in $f$-Electron Compounds, Phys. Rev. Lett. 81, 3531 (1998).

[60] A. Coldea and P. Reiss, Signatures of a quantum Griffiths phase close to an electronic nematic quantum phase transition, 10.5287/bodleian:z6676dkVw. 